\title{
A 3D CFD NUMERICAL STUDY OF THE BUBBLE GENERATION PROCESS INTO A BUBBLE T-JUNCTION GENERATOR AND ITS COMPARISON WITH EXPERIMENTAL DATA: PART I
}

\author{
S. Arias ${ }^{1}$ and A. Montlaur ${ }^{1,2}$ \\ ${ }^{1}$ Escola d'Enginyeria de Telecomunicació i Aeroespacial de Castelldefels, Universitat Politècnica de \\ Catalunya - BarcelonaTech \\ c/ Esteve Terradas 5, 08860, Castelldefels (Barcelona), Spain \\ e-mail: santiago.arias@upc.edu, adeline.de.montlaur@upc.edu \\ ${ }^{2}$ Laboratori de Càlcul Numèric, Universitat Politècnica de Catalunya, www-lacan.upc.edu
}

Keywords: Two-phase flows, Bubble generation, T-junction, Numerical simulation, Computational Fluid Dynamics (CFD), Volume of Fluid

\begin{abstract}
This work presents a $3 D$ numerical study of the bubble generation process into a bubble generator obtained with the commercial Computational Fluid Dynamics solver ANSYS Fluent v15.0.7, and its comparison with experimental data reproducing the same conditions [1]. The bubble generator is formed by two perpendicular capillaries in which liquid and gas are injected at perpendicular directions into a $1 \mathrm{~mm}$ internal diameter capillary T-junction with a total length of $10 \mathrm{~mm}$. The fluids used in experiments and CFD simulations are air and water, both of them considered incompressible and isothermal, at a room temperature of $25^{\circ}$. A total of 23 different cases are studied for different injection conditions, and results between numerical simulations and experiments are compared.

In this first part of the analysis, we focus on the flow pattern regimes and the dynamics of the bubble generation process. In addition to the new numerical simulations presented here, a new model has been used to predict the bubble generation frequency and tested with both experimental and numerical data. Results on bubble generation frequency are also presented by means of the non-dimensional Strouhal number. Same types of patterns, bubble and slug flow regimes, are obtained in simulations and experiments. In order to perform an exhaustive validation and comparison of numerical simulations with experimental data, several parameters have been selected: bubble velocity, volumetric void fraction, bubble generation frequency, Strouhal number and bubble equivalent diameter. Numerical simulations agree qualitatively, but not always quantitatively, with experimental results.
\end{abstract}




\section{INTRODUCTION}

Capillary gas-liquid two-phase flow occurs in increasingly more modern industrial applications and in particular in space-based systems. Two-phase systems present an improvement in performance as well as significant reductions in weight in different fields, such as power generation and life support systems, with respect to one-phase systems. A full understanding of the behaviour of the gas-liquid interfaces and flow characteristics, such as flow patterns and flow patterns transitions, is thus a major necessity in the development of these technologies.

Numerous experimental studies have been made on the generation of bubbles and droplets in T-junction bubble generators as the one studied in this paper [2, 3]. In the recent past, different Computational Fluid Dynamics (CFD) methods have also been used in order to study similar devices. A commercial CFD package was used to simulate the squeezing regime during the bubble formation in a T-junction microchannel [4], focusing on studying the effects of pressure, surface tension and shear stress action on the gas thread. CFD modelling aspects of internal circulation and slug flow generation have been discussed, focusing on the slug flow formation in a $120^{\circ}$ Y-junction and the velocity profiles inside the slug [5]. A numerical investigation by means of a phase-field model of the breakup dynamics of streams of immiscible fluids in a microfluidic T-junction was carried out [6], where three regimes of formation of droplets (squeezing, dripping and jetting) were identified and studied.

More recently, a fluid dynamics numerical study of the formation of mini-bubbles in a 2D T-junction was presented [1], and results on the behaviour of bubble velocity, void fraction, bubble generation frequency and characteristic lengths were obtained and compared to experimental data. Also using a two-dimensional numerical model with Volume of Fluid method (VOF), alternating droplet formation, with applications such as nanoparticle synthesis, hydrogel bead generation, and cell transplantation in biomedical therapy, was studied [7]. The twodimensional bubble formation process was analysed in detail by focusing on the effects of three main parameters, capillary number, contact angle and the gas-liquid flow rate ratio [8]. As for 3D simulations, droplet formation in T-junction microchannels was studied using the Lattice Boltzmann method [9] and simulations in heat exchangers using the VOF method were performed [10].

The paper presented here is a continuation of a previous study [1], completing it and extending it to 3D. Some parameters are now studied in more depth, such as the bubble generation frequency, a new model of frequency being developed here, and new values of interest are presented, such as the bubble velocity, volume void fraction, Strouhal number, bubble volume and the equivalent diameter. Section 2 presents the T-junction problem statement. Section 3 summarises the methodology used in the experimental study, following the one used in [1], as well as the hypotheses used in the numerical simulations and their validations. Section 4 exposes the results of the numerical simulations and compares them to the experimental ones. Finally, conclusions are drawn in section 5 .

\section{PROBLEM STATEMENT}

The presented study aims to simulate a bubble generator in which liquid and gas are injected at perpendicular directions into a $1 \mathrm{~mm}$ internal diameter capillary T-junction with a total length of $10 \mathrm{~mm}$, see figure 1 . In order to compare numerical simulations with realistic results, CFD simulations have been carried out in the most similar conditions possible to the experiments. The fluids used in experiments and CFD simulations are air and water (incompressible, isothermal, at constant room temperature of $25^{\circ} \mathrm{C}$ ). Standard physical values are assumed for 
air (density $\rho_{G}=1.225 \mathrm{~kg} / \mathrm{m}^{3}$, viscosity $\mu_{G}=10^{-5}$ Pa s) and water (density $\rho_{L}=10^{3} \mathrm{~kg} / \mathrm{m}^{3}$, viscosity $\left.\mu_{L}=10^{-3} \mathrm{~Pa} \mathrm{~s}\right)$ and surface tension at the gas-liquid interface $(\sigma=0.072 \mathrm{~N} / \mathrm{m})$.

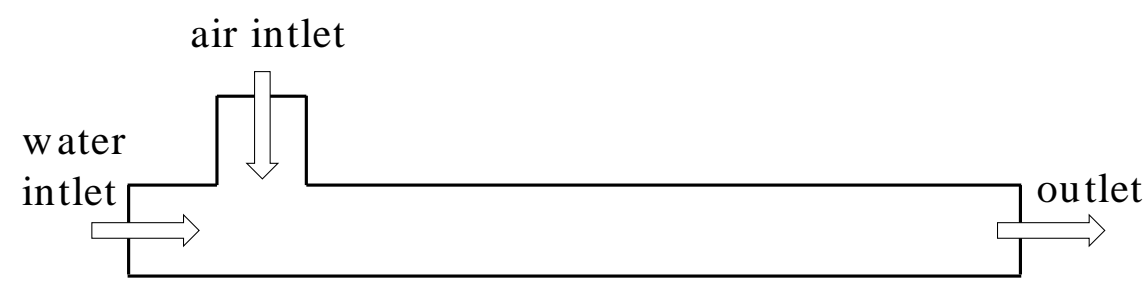

Figure 1: Sketch of T-junction.

The experimental setup used in this study [11, 12] can control the gas and liquid volumetric flow rates ( $Q_{G}$ and $Q_{L}$, respectively) in a very accurate way. Results are presented as a function of the gas and liquid superficial velocities defined as $U_{S G}=Q_{G} / A$ and $U_{S L}=Q_{L} / A$, respectively, where $A$ is the capillary cross-sectional area.

Constant gas and liquid superficial velocities $\left(Q_{G}=0.062-0.473 \mathrm{~m} / \mathrm{s}\right.$ and three different $Q_{L}=0.106,0.318,0.531 \mathrm{~m} / \mathrm{s}$, respectively) were considered in each experimental run. A total of 23 experiments were compared with the results obtained in numerical simulations. The same injection conditions were used in the simulations. Additionally, three more simulations corresponding to the value $U_{S G}=0.025 \mathrm{~m} / \mathrm{s}$ (and $Q_{L}=0.106,0.318,0.531 \mathrm{~m} / \mathrm{s}$ ) were conducted to obtain higher precision at very low superficial gas velocity than provided by experiments.

According to the Bond number, gravity played a smaller role than surface tension in the bubble generation process $\left(\rho_{L} g \phi_{c}^{2} / \sigma=0.139\right.$, where $g$ is the gravitational acceleration and $\phi_{c}$ the capillary internal diameter), resulting in the irrelevance of the channel orientation [13]. Therefore, the present analysis can be considered gravity independent and assuming $g=0 \mathrm{~m} / \mathrm{s}^{2}$ becomes a valid approach for numerical simulations.

In the considered experimental conditions, the formation and detachment of bubbles is mainly dominated by the competition between the capillary and the liquid drag forces, which can be evaluated by the Weber number $\left(W e_{G}=\rho_{G} \phi_{c} U_{G}{ }^{2} / \sigma\right.$, where $U_{G}$ is the gas (bubble) velocity). Capillary forces overcome the inertial forces when the criterion $W e_{G}<2$ is accomplished [14], and only bubble and slug flow regimes are expected to be observed in those circumstances. In our experiments, $W e_{G}$ ranges from $4.3 \times 10^{-4}$ to $2.2 \times 10^{-2}\left(U_{G}=0.159-1.143 \mathrm{~m} / \mathrm{s}\right.$, see section 4.1), corresponding to the surface tension controlled region.

The Reynolds number, based on the average mixture superficial velocity $\left(U_{M}=U_{G}+U_{L}\right)$ and defined as $R e_{M}=\rho_{L} \phi_{c} U_{M} / \mu_{L}$, ranged from 167 to $1000\left(U_{M}=0.167-1 \mathrm{~m} / \mathrm{s}\right.$ in experiments), and hence we assume laminar conditions.

Having used the same fluids physical properties as well as the same values of gas and liquid superficial velocities in experiments and simulations, the bubble generation behaviour in numerical simulations is expected to be similar to the one observed in experiments.

\section{METHODOLOGY}

\subsection{Experiments}

Experiments were performed at constant liquid superficial velocity while increasing the gas superficial velocity. The generation of bubbles was observed to be regular and periodic for all gas and liquid superficial velocities considered in this work. Images were taken with a highspeed camera at 4000 f.p.s. or each pair of $U_{S G}$ and $U_{S L}$ values. These images were classified 
into bubble or slug flow regimes [15]. Bubble generation frequency was measured by counting the total number of bubbles over a period of time larger than the time required to form a single bubble (typically three orders of magnitude smaller). The bubble velocity was measured directly over the calibrated images, by taking into account the displacement of the foremost part of a single bubble and the time taken to carry out such a movement.

\subsection{Numerical simulations}

\subsubsection{Flow solver}

All numerical simulations were carried out with a commercial CFD solver, ANSYS Fluent v15.0.7. The VOF model was used to model the two immiscible fluids by solving a single set of momentum equations and tracking the volume fraction of each fluid throughout the domain. In each control volume, the volume fractions of both phases sum to unity.

The tracking of the interface between the phases is accomplished by the solution of a continuity equation for the volume fraction of one of the phases [16]. For the $q^{\text {th }}$ phase, this equation has the following form:

$$
\frac{1}{\rho_{q}}\left[\frac{\partial}{\partial t}\left(\alpha_{q} \rho_{q}\right)+\nabla \cdot\left(\alpha_{q} \rho_{q} \mathbf{v}_{\mathbf{q}}\right)=\sum_{\mathbf{p}=\mathbf{1}}^{\mathbf{n}}\left(\dot{\mathbf{m}}_{\mathbf{p q}}-\dot{\mathbf{m}}_{\mathbf{q p}}\right)\right]
$$

where $\rho_{q}, \alpha_{q}$ and $\mathbf{v}_{\mathbf{q}}$ are the density, volume void fraction and velocity of phase $q$, respectively, and $\dot{m}_{q p}$ is the mass transfer from phase $q$ to phase $p$ and $\dot{m}_{p q}$ is the mass transfer from phase $p$ to phase $q$. The volume fraction equation is not solved for the primary phase; the primary-phase volume fraction is computed based on the following constraint: $\sum_{q=1}^{n} \alpha_{q}=1$.

The fields for variables and properties are shared by the phases and represent volumeaveraged values, as long as the volume fraction of each of the phases is known at each location. Thus the variables and properties in any given cell are either purely representative of one of the phases, or representative of a mixture of the phases, depending on the volume fraction values. That is, if the water volume fraction in the cell is denoted as $a_{w}$, then three conditions are possible. If $a_{w}=0$, the cell is empty of water, if $a_{w}=1$, the cell is full of water, and if $0<a_{w}<1$, the cell contains the interface between water and air [16]. Based on the local value of $a_{w}$, the appropriate properties and variables are assigned to each control volume within the domain.

An explicit formulation is used for the VOF model, with a Courant number of 0.25 , and the PRESTO! (PREssure STaggering Option) scheme is used for pressure interpolation. This scheme uses the discrete continuity balance for a staggered control volume about the face to compute the staggered pressure. This procedure is similar in spirit to the staggered-grid schemes used with structured meshes [16]. For the volume fraction spatial discretisation scheme, the geometric reconstruction scheme, which represents the interface between fluids using a piecewiselinear approach is used. In Fluent this scheme is the most accurate and is applicable for general unstructured meshes. The geometric reconstruction scheme is generalised for unstructured meshes from the work of [17].

The VOF model also includes here the effects of surface tension along the interface between each pair of phases. The surface tension model in Fluent is the continuum surface force model proposed by [18]. With this model, the addition of surface tension to the VOF calculation results in a source term in the momentum equation. As commented in section 2, a surface tension of $0.072 \mathrm{~N} / \mathrm{m}$ is applied.

The following boundary conditions were set for the numerical simulations. All walls are 


\begin{tabular}{ccccccc}
\hline number of elements & $U_{G}[\mathrm{~m} / \mathrm{s}]$ & $\epsilon_{U_{G}}[\%]$ & $f[1 / \mathrm{s}]$ & $\epsilon_{f}[\%]$ & $V_{B}\left[\times 10^{-10} \mathrm{~m}^{3}\right]$ & $\epsilon_{V_{B}}[\%]$ \\
\hline 220000 & 0.649 & 7.1 & 266.7 & 14.7 & 7.31 & 8.1 \\
413000 & 0.625 & 3.1 & 238.1 & 2.4 & 7.82 & 1.6 \\
879000 & 0.606 & - & 232.6 & - & 7.95 & - \\
\hline
\end{tabular}

Table 1: Study of mesh convergence for three meshes and three independent parameters of study.

treated as no-slip smooth walls. A wall adhesion angle in conjunction with the surface tension model is also applied in the VOF model [18]. Rather than imposing this boundary condition at the wall itself, the contact angle that the fluid is assumed to make with the wall is used to adjust the surface normal in cells near the wall [16]. This so-called dynamic boundary condition results in the adjustment of the curvature of the surface near the wall. The contact angle is the angle between the wall and the tangent to the interface at the wall. A contact angle of $0^{\circ}$ was set up as wall adhesion (hydrophilic condition) on the horizontal walls, and of $25^{\circ}$ on the vertical walls, based on experimental data [1].

Water and air inlets are considered as velocity inlets, with the corresponding fluid velocity in each case of study, namely $U_{S L}$ and $U_{S G}$. At the water inlet, the fraction of water is considered as 1 and at the air inlet, the fraction of air is the one set to 1 . The outlet of the T-junction is set up as a pressure outlet.

An operating pressure of $101325 \mathrm{~Pa}$ is set up at a point close to the air inlet, that is, at a point where there will always be only air. Finally the gravity value is set to 0 , as explained in section 2 .

\subsubsection{Mesh}

The computational mesh was composed of 413000 elements. An inflation mesh comprising 15 layers was used along all the walls, with a first layer height of $0.005 \mathrm{~mm}$. A body sizing of $0.045 \mathrm{~mm}$ was used in all the domain. The resulting mesh is a mix of hexahedral (close to the walls) and tetrahedral (in the core of the domain) elements. The hex dominant option has been chosen, resulting in a fewer number of elements than if mainly tetrahedral elements had been considered. Mesh independence is presented in section 3.2.3.

A first-order implicit scheme is used for the transient formulation. Time step independence is also presented in section 3.2.3. In order to obtain a converged solution, the calculation was always continued until at least 7 or 8 bubbles of air were generated.

\subsubsection{CFD validation}

The mesh convergence is first checked. Three meshes are considered: the coarsest one of 220000 elements, the second one obtained by multiplying the body sizing and first layer height of the boundary layer by 0.75 , of 413000 elements, and the third one, obtained by multiplying again the mesh dimensions by 0.75 , of 879000 elements. Three independent parameters of study are shown, which will be later used in section 4 , the bubble velocity $U_{G}$, the bubble frequency $f$ and the bubble volume $V_{B}$. Absolute values are shown, as well as the percentages of error with respect to the finest mesh, $\epsilon$, in table 1 , for $U_{S L}=0.318 \mathrm{~m} / \mathrm{s}$ and $U_{S G}=0.242 \mathrm{~m} / \mathrm{s}$.

These results show that for the 413000 element mesh, errors due to the mesh remain within a relatively small margin $(\leq 3.1 \%)$; this is thus the mesh that has been chosen for the numerical study. 


\begin{tabular}{ccccccc}
\hline$\Delta t[\mathrm{~s}]$ & $U_{G}[\mathrm{~m} / \mathrm{s}]$ & $\epsilon_{U_{G}}[\%]$ & $f[1 / \mathrm{s}]$ & $\epsilon_{f}[\%]$ & $V_{B}\left[\times 10^{-10} \mathrm{~m}^{3}\right]$ & $\epsilon_{V_{B}}[\%]$ \\
\hline $10 \times 10^{-6}$ & 0.633 & 1.9 & 243.9 & 3.5 & 8.04 & 5.0 \\
$5 \times 10^{-6}$ & 0.625 & 0.6 & 238.1 & 1.1 & 7.82 & 2.1 \\
$2.5 \times 10^{-6}$ & 0.621 & - & 235.6 & - & 7.66 & - \\
\hline
\end{tabular}

Table 2: Study of time convergence for three $\Delta t$ and three independent parameters of study.

Next, the influence of the time step has been studied considering the chosen mesh of 413000 elements. Three values of $\Delta t$ are considered: $\Delta t=2.5 \times 10^{-6} \mathrm{~s}, \Delta t=5 \times 10^{-6} \mathrm{~s}$ and $\Delta t=10 \times 10^{-6} \mathrm{~s}$. Again absolute values of velocity, bubble frequency and volume are shown in table 2, as well as the percentages of error with respect to the smallest $\Delta t$, for $U_{S L}=0.318$ $\mathrm{m} / \mathrm{s}$ and $U_{S G}=0.242 \mathrm{~m} / \mathrm{s}$.

Table 2 shows that the three values of $\Delta t$ lead to similar values (all errors $\leq 5.0 \%$ ), smaller for the value of $\Delta t=5 \times 10^{-6} \mathrm{~s}(\leq 2.1 \%)$ than for the value $\Delta t=10 \times 10^{-6} \mathrm{~s}$. Therefore, the middle value, $\Delta t=5 \times 10^{-6} \mathrm{~s}$, has been chosen for the rest of the CFD study.

\subsubsection{CFD metrics}

Figure 2 shows an example of data obtained as a post-process of the CFD simulations. It shows the propagation of the bubble, through the fraction of air at two cross-sections of the domain located at 7 and $8 \mathrm{~mm}$ from the beginning of the tube, as a function of time. This allows to calculate the bubble frequency $f$, as the inverse of the time $T_{f}$ between two bubbles at the cross-section, and its velocity $U_{G}$, as the distance between two cross-sections (here 7 and $8 \mathrm{~mm}$ ) divided by the time $T_{S}$ needed for the bubble to travel this distance. Its volume $V_{B}$ is also calculated from this figure, integrating the total fraction of air of a bubble over time $A_{B}$ and multiplying by its velocity and by the area of a $1 \mathrm{~mm}$ diameter circle. The volume void fraction, $\alpha$, was estimated for both the experiments and the CFD simulations as the ratio $U_{S G} / U_{G}$ [19].

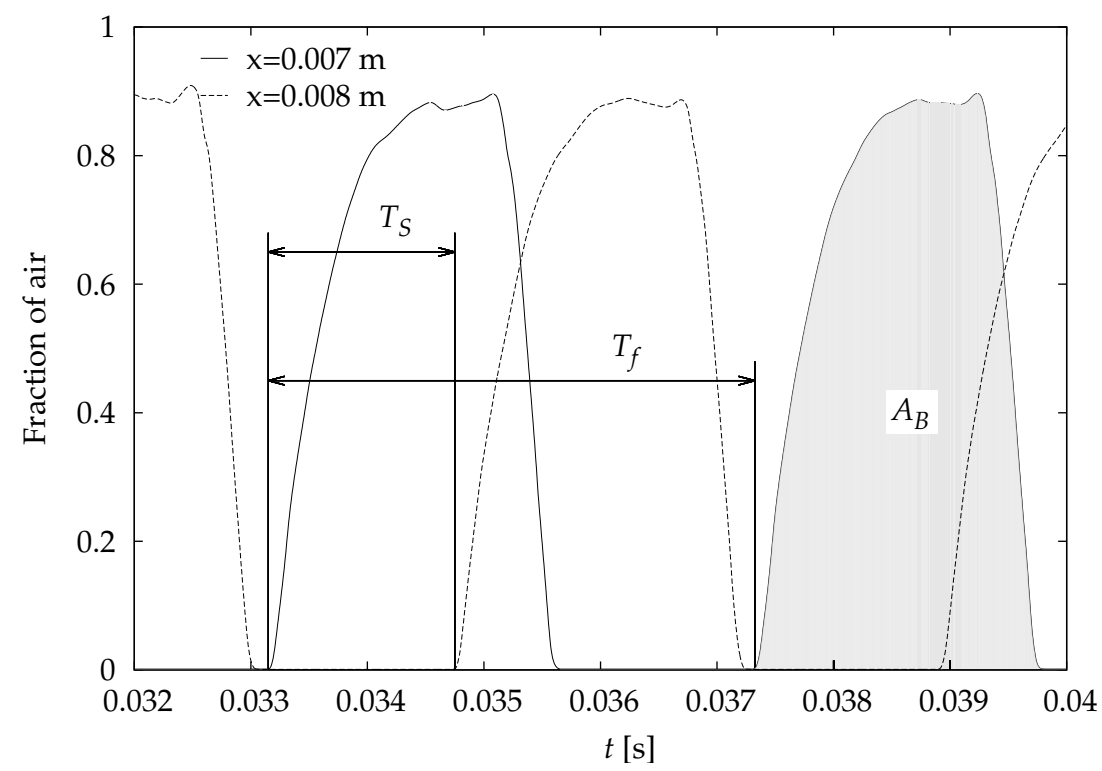

Figure 2: Example of CFD post-processed results from the graph of fraction of air as a function of time, for $U_{S L}=0.318 \mathrm{~m} / \mathrm{s}$ and $U_{S G}=0.242 \mathrm{~m} / \mathrm{s}$. 


\section{RESULTS AND DISCUSSION}

A set of numerical simulations are compared with experimental data. Numerical simulations satisfactorily reproduce trains of bubbles as the ones obtained in experiments. As expected, only two flow patterns, the bubble and the slug flow regimes, were obtained in the simulations and in the experiments [13, 14, 20]. In both flow regimes the gas phase is dispersed into the liquid phase. Spherical or almost spherical bubbles with a diameter smaller than the capillary diameter were classified as bubble flow. Larger bubbles were classified as slug flow [15].

Trains of bubbles are shown in figs. 3 ,5 at the same stage of formation, which do not correspond to the same times as detailed in section 4.2. In contrast with a previous study [1], no artificial pinch-off mechanism has been used in numerical simulations to detach bubbles. Bubbles were generated, both in experiments and in numerical simulations, with high regularity and small size dispersion. Fig. 3 corresponds to the formation of a bubble belonging to the bubble flow regime. In this figure, bubbles are not exactly spherical. This is a consequence of the liquid drag force, which noticeably deforms bubbles longitudinally. Figs. 4 and 5 show two examples of slug flow regime, the former being known as short Taylor bubble and the latter as middle Taylor bubble [21]. Bubbles from the slug flow regime are characterised by having a bullet-shaped body, rounded at the front part and mainly flattened at the rear of it. In every case, bubbles have an elongated rear part just after the detachment, this elongated shape being a remnant of the squeezed gas thread that was connecting the new bubble with the single-phase gas. From that moment, the effect of the surface tension tries to minimise the total surface of the bubble, which results in the vibration of the bubble surface until it stabilises. It can be seen that numerical simulations correctly reproduce the elasticity and fluctuation of the gas-liquid interface during the bubble detachment stage described here.

Next sections focus in a more exhaustive validation and comparison of numerical simulations with experimental data. Several parameters are selected to perform such a validation, namely, bubble velocity, volumetric void fraction, bubble generation frequency and bubble equivalent diameter.

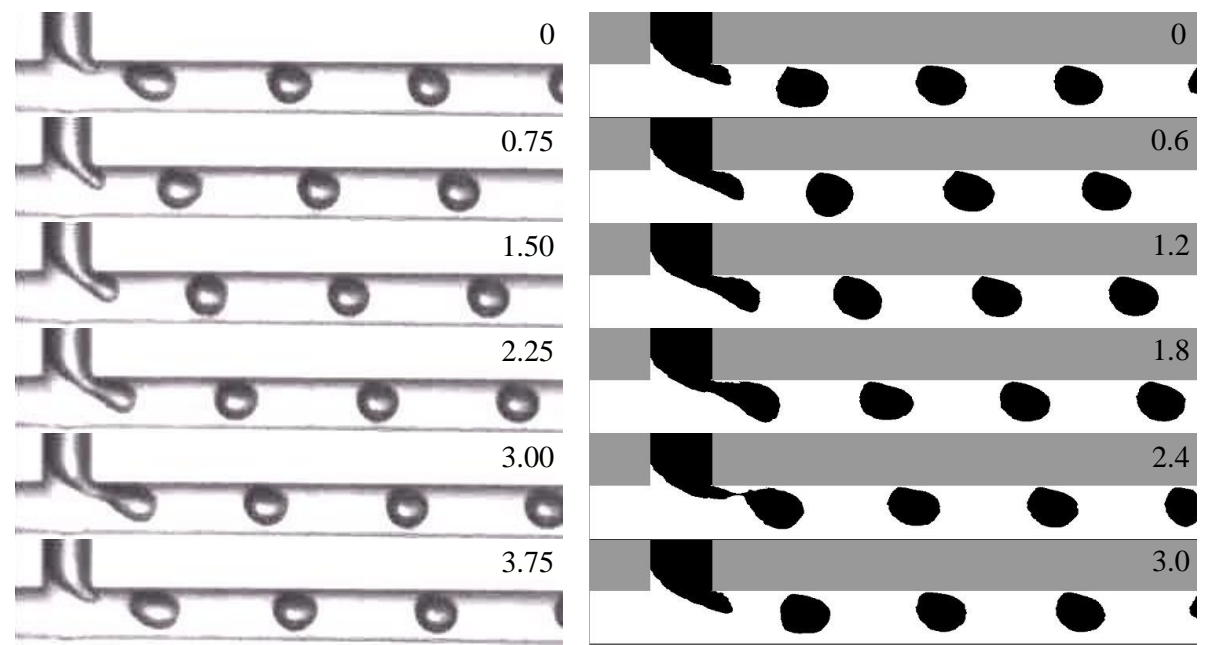

Figure 3: Bubble flow in (left) experiments and (right) numerical simulations. $U_{S L}=0.531 \mathrm{~m} / \mathrm{s}$ and $U_{S G}=$ $0.110 \mathrm{~m} / \mathrm{s}$. Time $(\mathrm{ms})$ is indicated in the upper right corner. 

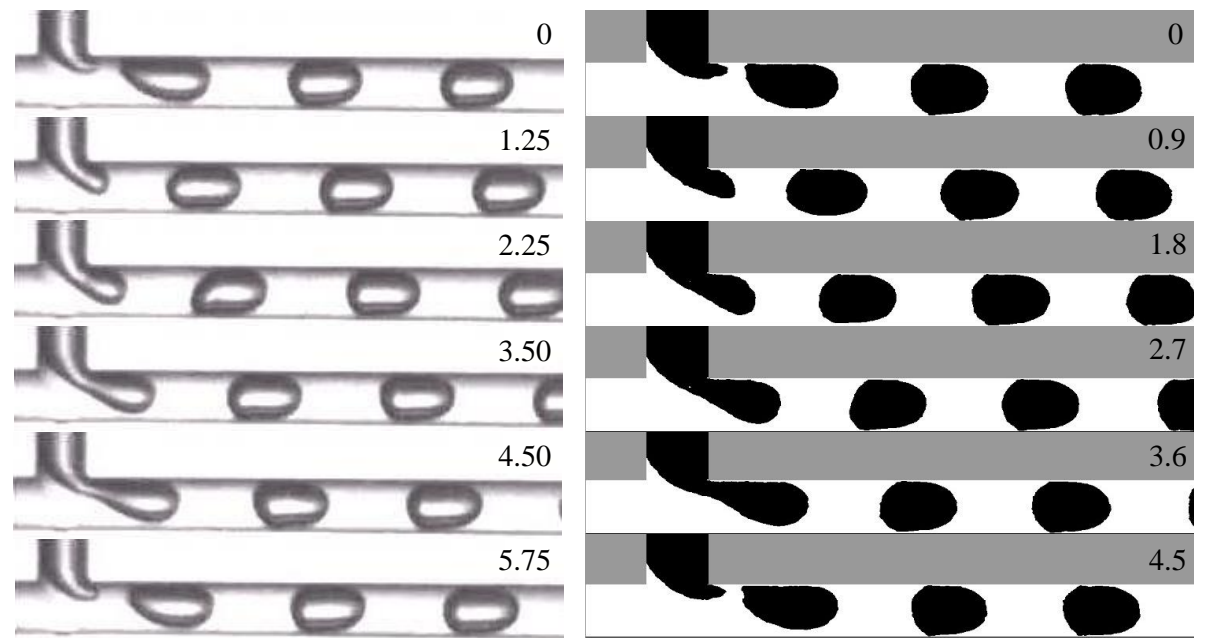

Figure 4: Slug flow in (left) experiments and (right) numerical simulations. $U_{S L}=0.318 \mathrm{~m} / \mathrm{s}$ and $U_{S G}=$ $0.182 \mathrm{~m} / \mathrm{s}$. Time $(\mathrm{ms})$ is indicated in the upper right corner.
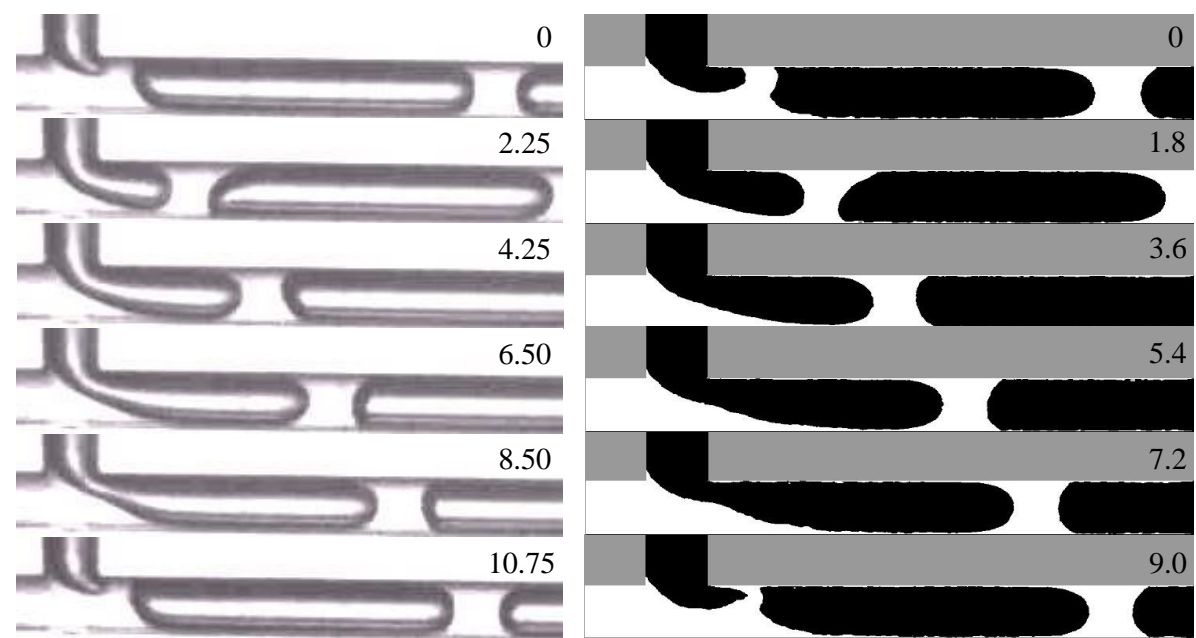

Figure 5: Slug flow close to the slug-churn transition in (left) experiments and (right) numerical simulations. $U_{S L}=0.106 \mathrm{~m} / \mathrm{s}$ and $U_{S G}=0.471 \mathrm{~m} / \mathrm{s}$. Time $(\mathrm{ms})$ is indicated in the upper right corner.

\subsection{Bubble velocity and void fraction}

Fig. 6 shows the bubble velocity as a function of the mixture superficial velocity. Data follow a linear tendency, and $U_{G}$ increases when increasing $U_{M}$, as expected [19]. As a consequence, the bubble velocity was fitted with the drift-flux model [24], which under the hypothesis of negligible gravity levels can be written as [1, 19, 25]:

$$
U_{G}=C_{0} U_{M}=C_{0}\left(U_{S G}+U_{S L}\right),
$$

where $C_{0}$ is the void fraction distribution coefficient, which considers both the effect of the non-uniform velocities of the gas and liquid phases as well as the void profiles through the flow capillary. The value of $C_{0}$ has been calculated as the slope of the straight line of best fit plotted in figure 6. A value of 1.10 was found for the experimental void fraction distribution coefficient. Numerical simulations correctly reproduced the same behaviour than the one observed in the experiments. $C_{0}$ was found to be 1.15 in numerical simulation, $4.5 \%$ greater than in the experiments. The percentage of error with respect to the experimental data range from $0.8 \%$ to $19.3 \%$, 
for the smallest value of $U_{S G}$ and $U_{S L}$, with an average in difference of $5.4 \%$. Both values of $C_{0}$ agree with previous results of gas and liquid mixtures flowing in minichannel reported in the related literature [1, 25]. Moreover, being greater than 1 , both values of $C_{0}$ prove that bubbles moves faster than the mixture, which is due to the fact that bubbles move mainly through the centreline of the capillary.

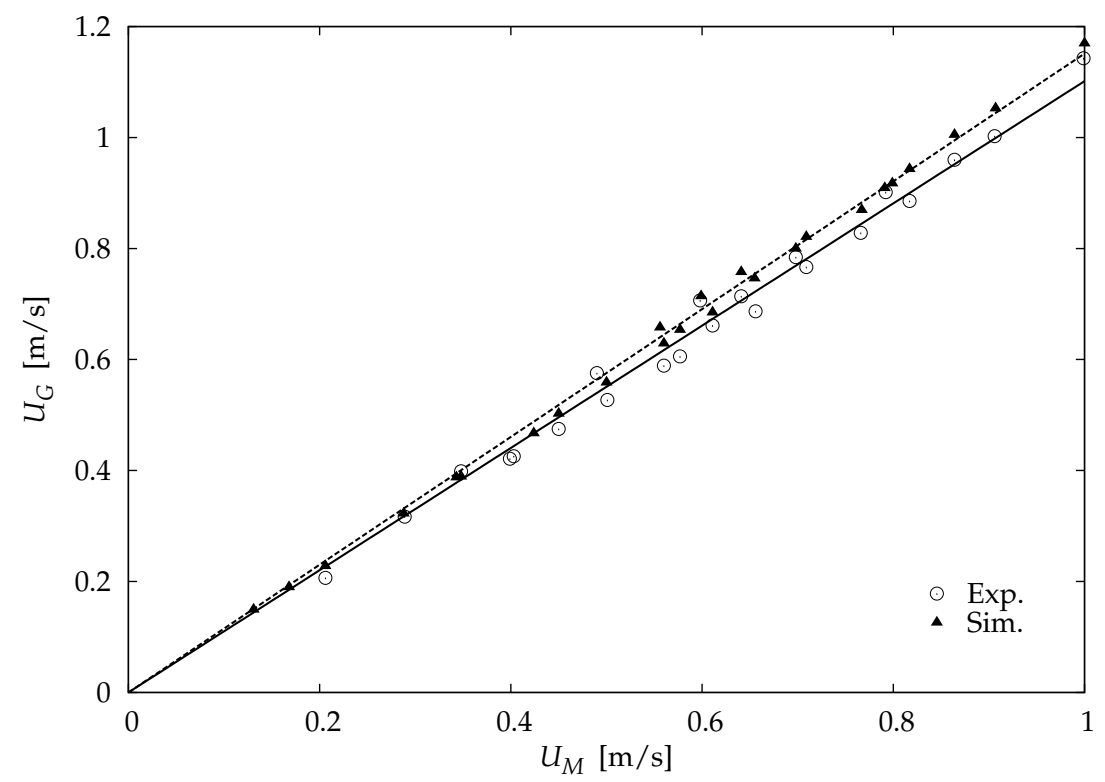

Figure 6: Bubble velocity as a function of the mixture superficial velocity. Empty symbols: experimental data, solid symbols: numerical data. Lines: fitting of the data by using Eq. 2, with $C_{0}=1.10$ for experimental data (solid line) and $C_{0}=1.15$ for numerical simulation (dashed line).

Combining Eq. 2 and the expression $\alpha=U_{S G} / U_{G}$, an analytical prediction of the volume void fraction as a function of the gas and liquid superficial velocities can be found:

$$
\alpha=\frac{1}{C_{0}\left(1+\frac{U_{S L}}{U_{S G}}\right)}
$$

Figures 7 shows the volume void fraction as a function of the ratio of the gas and liquid superficial velocities. The prediction of $\alpha$ provided by Eq. 3 has also been plotted using the corresponding value of $C_{0}$ in each case. Both experimental and numerical data fit well to their respective predictions. The values of void fraction were found to be slightly smaller in the numerical simulations, coherently with their previously observed greater values of velocity. A minimum and maximum relative error of $0.8 \%$ and $15.5 \%$, respectively, and an average value of $5.0 \%$ were found. 3D numerical simulations proved to be useful by providing information that was not available from the experiments (two points below the value $U_{S G} / U_{S L}=0.1$ ).

\subsection{Bubble generation frequency}

Figure 8 shows the bubble generation frequency, $f$, versus the superficial gas velocity for both experiments and numerical simulations. Data corresponding to three different superficial liquid velocity has been plotted. Three additional points corresponding to the superficial gas velocity of $0.025 \mathrm{~m} / \mathrm{s}$, which are not present in the experimental data, were also simulated in order to enrich the knowledge about the behaviour of bubble generation frequency 


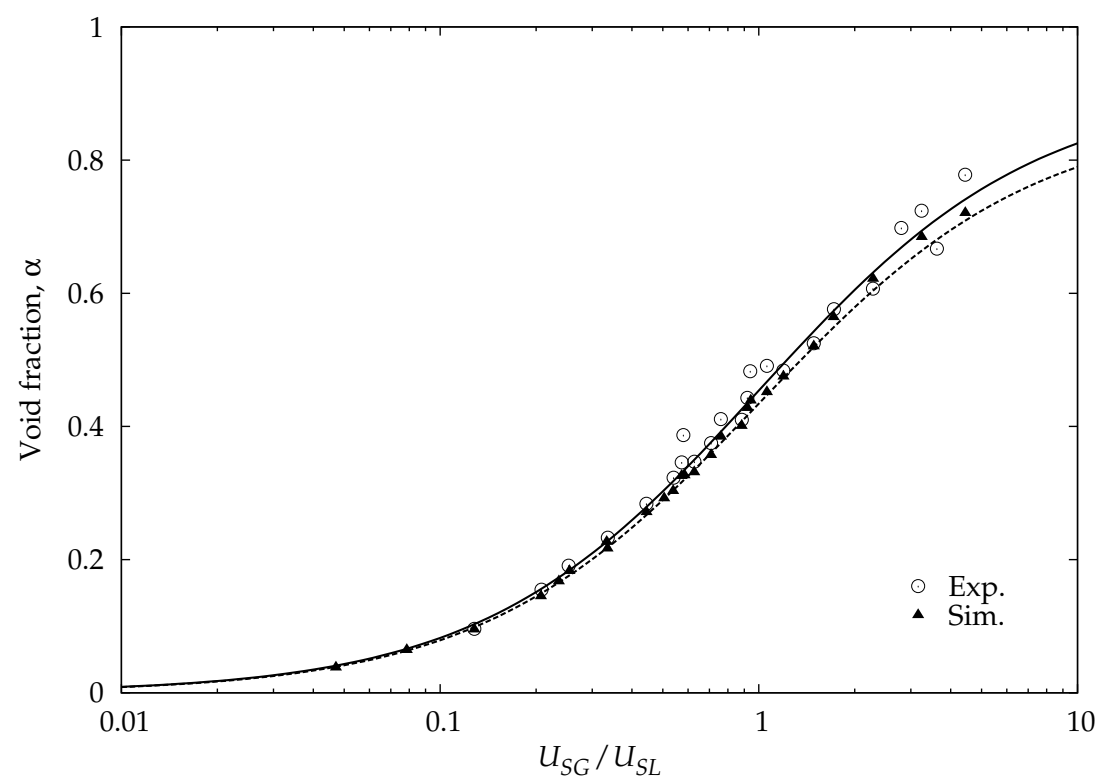

Figure 7: Void fraction as a function of the ratio of the gas and liquid superficial velocities. Empty symbols: experimental data, solid symbols: numerical data. Lines: theoretical prediction given by Eq. 3 , with $C_{0}=1.10$ for experimental data (solid line) and $C_{0}=1.15$ for numerical simulation (dashed line).

At very low gas flow rate, $f$ follows a close-to-linear tendency (the linear regime) that progressively curves when increasing $U_{S G}$ until reaching a saturation value, $f_{\text {sat }}$, that remains constant thereafter (the saturation regime). Both behaviours (and regimes) were previously reported [11, 12].

Figure 8 shows how $f$ approaches its corresponding saturated value for the three different values of $U_{S L}$. The saturation frequency increases when increasing $U_{S L}$, which is a consequence of the corresponding increment of the liquid drag force, the main force in charged of detaching bubbles [22, 23]. Therefore, larger liquid drag forces are expected to produce smaller bubbles resulting in larger frequency values [11, 12]. This effect also explains why the linear slope at the origin, $a_{0}$, increases when increasing the liquid superficial velocity (see table 3 ).

\begin{tabular}{ccccc}
\hline & \multicolumn{2}{c}{ Experiments } & \multicolumn{2}{c}{ Simulations } \\
$U_{S L}[\mathrm{~m} / \mathrm{s}]$ & $a_{0}\left[\mathrm{~m}^{-1}\right]$ & $f_{\text {sat }}[1 / \mathrm{s}]$ & $a_{0}\left[\mathrm{~m}^{-1}\right]$ & $f_{\text {sat }}[1 / \mathrm{s}]$ \\
\hline 0.106 & 616.4 & 99.7 & 856.6 & 114.5 \\
0.318 & 2033.9 & 228.4 & 2211.6 & 282.4 \\
0.531 & 4483.6 & 376.3 & 6426.8 & 392.0 \\
\hline
\end{tabular}

Table 3: Values of $a_{0}$ and $f_{\text {sat }}$ obtained when fitting the experimental and numerical data by using Eq. 4 .

As shown in figure 8, numerical simulations satisfactorily describe the behaviour of $f$ observed in experiments. Numerical simulations agree qualitatively, but not quite quantitatively, with experimental results, providing values in accordance but always larger than the experimental ones. These quantitative differences, calculated as the percentages of error with respect to the experimental data, range from $3.5 \%$ (within the margin of error of the mesh) to $34 \%$ ( $U_{S G}=0.025 \mathrm{~m} / \mathrm{s}$ and $U_{S L}=0.106 \mathrm{~m} / \mathrm{s}$ ) with an average value of $17 \%$. One possible explanation of this disagreement, could be that numerical simulations do not adequately resolve the necking region during the bubble pinch-off, leading to an inaccurate calculation of the surface 


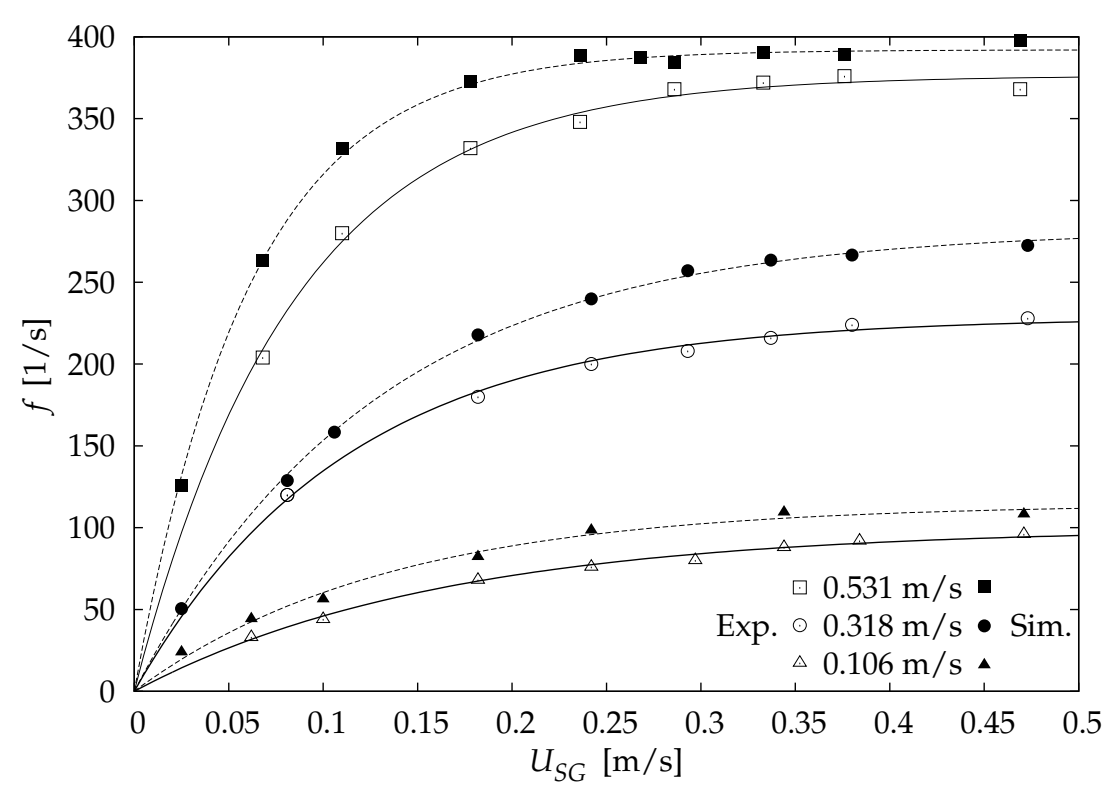

Figure 8: Bubble frequency as a function of the superficial gas velocity for three different superficial liquid velocities. Empty symbols: experimental data, solid symbols: numerical data. Solid lines: fitting of the experimental data by using Eq.4. dashed lines: fitting of the numerical data by using Eq.4

tension forces and to a premature pinch-off of the bubble. Future work should focus especially on this point with a more detailed pinch-off study.

A simple exponential model, which fits well and smoothly both experimental and numerical data, is used to fit the values of the bubble generation frequency in figure 8 ;

$$
f=f_{\text {sat }}\left(1-e^{\left(-\frac{a_{0}}{f_{s a t}} U_{S G}\right)}\right)
$$

Only two parameters are considered in this model, the initial slope $a_{0}$ of the linear regime (i.e. slope when $U_{S G}=0$ ), and the value of the saturation frequency $f_{\text {sat }}$. Eq. 4 is consistent with the experimental observations regarding the existence of a linear and a saturation regime. Assuming a regular generation of bubbles in size, the gas flow rate injected into the capillary must be equal to the bubble volume times the frequency, $Q_{G}=V_{B} f$, which leads to $f=A U_{S G} / V_{B}$. Matching this last expression with the first-order Taylor series expansion of Eq. 4, $f_{0}=a_{0} U_{S G}$, shows that $a_{0}=A / V_{B}$. The initial slope of the linear regime corresponds to the maximum value of the ratio $f / U_{S G}$ for each given $U_{S L}$. Then, $a_{0}$ is related to the minimum bubble volume that can be generated for each given $U_{S L}$, that is, $\left.V_{B}\right|_{\min }=A / a_{0}$. As a consequence, the greater the initial slope at the linear regime, the greater the liquid drag force and the smaller the minimum volume of bubble that can be generated for any $U_{S L}$. The saturation frequency is related in turn to the minimum time required to form a bubble, marking a limiting scale for the bubble generation process [11].

Table 3 summarises the values obtained for $a_{0}$ and $f_{\text {sat }}$ when fitting both the experimental and numerical data by using Eq. 4. In every case, these values of both $a_{0}$ and $f_{\text {sat }}$ were found to be of the same order of magnitude but larger in the numerical simulations. This result shows again that the bubble generation process was faster in the simulations than in the experiments, resulting in larger frequencies and smaller bubbles size in the numerical simulations.

The non-dimensional Strouhal number, St, is used as a normalisation of the bubble generation frequency. $S t$ is defined here by means of the gas velocity as $S t=f \phi_{c} / U_{G}$. Two 
linear and independent tendencies with respect to the volume void fraction can be found for $S t$ at small and larger values of $\alpha$. For small values of $\alpha$ (linear regime), St can be written as $S t=\bar{a}_{0} \alpha$ ( $a_{0}$ being normalised with $\phi_{c}$ ), by taking into account the first-order Taylor series expansion of eq. 4. For larger values of $\alpha$ (saturation regime), $S t$ can be written in turn as $S t=f_{\text {sat }} \phi_{c}\left(1-C_{0} \alpha\right) /\left(C_{0} U_{S L}\right)$, by using eq. 3 and the definition of $\alpha$. Being the ratio $f_{\text {sat }} \phi_{c} /\left(C_{0} U_{S L}\right)$ a constant value for each given liquid superficial velocity, $S t$ becomes proportional to $\left(1-C_{0} \alpha\right)$ in the saturation regime.

Fig. 9 shows the Strouhal number as a function of the volume void fraction. Eq. 3 has been rewritten to provide the change between variables $\left(U_{S G}\right.$ and $\left.\alpha\right)$ as $U_{S G}=C_{0} U_{S L} \alpha /\left(1-C_{0} \alpha\right)$. The same values of $C_{0}$ obtained in section 4.1 has been used in this figure. Eq. 4 is also used here to provide a prediction in fig. 9. Both linear tendencies, $S t \propto \alpha$ and $S t \propto\left(1-C_{0} \alpha\right)$ (in the linear and saturation regime, respectively), can be observed in the figure. The location of a crossover point between the linear and saturation regimes (for each $U_{S L}$ ) can be defined as the maximum value in this figure. Numerical simulations behave similarly to experimens. The percentage of error in $S t$ range from $0.3 \%$ to $32.6 \%$ with an average in difference of $11.9 \%$. Values of $S t$ are greater in simulations than in experiments, correspondingly to their bigger values of $f$. The increase in $U_{S L}$ also increases $S t$, as a consecuence again of the increase in $f$.

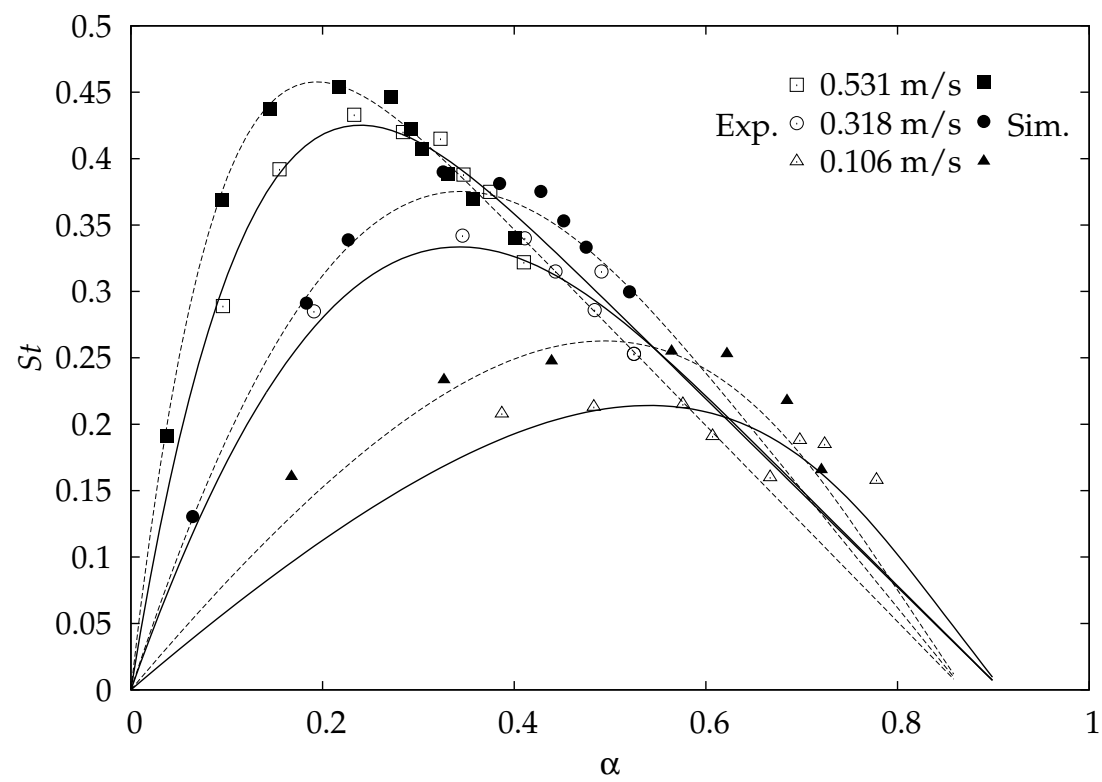

Figure 9: Strouhal number, St, as a function of the volume void fraction, $\alpha$, for three different superficial liquid velocities. Empty symbols: experimental data, solid symbols: numerical data. Solid lines: prediction of the experimental data by using Eq. 4 and 2 , dashed lines: prediction of the numerical data by using Eq. 4 and 2.

\subsection{Bubble volume and equivalent diameter}

Normalising the relationship $Q_{G}=V_{B} f$ with the cross section area times the capillary diameter, a non-dimensional expression of the bubble volume can be obtained:

$$
\bar{V}_{B}=\frac{U_{S G}}{f \phi_{c}} .
$$

The bubble volume was measured in the $3 \mathrm{D}$ numerical simulations. No direct measures of $V_{B}$ could be performed in the experiments. For that reason, figure 10 shows results only 
from numerical simulations. In that figure, the non-dimensional bubble volume is plotted as a function of the ratio $U_{S G} /\left(f \phi_{c}\right)$. Data fit well to the prediction of Eq. 5, which confirms that bubbles were generated with high regularity.

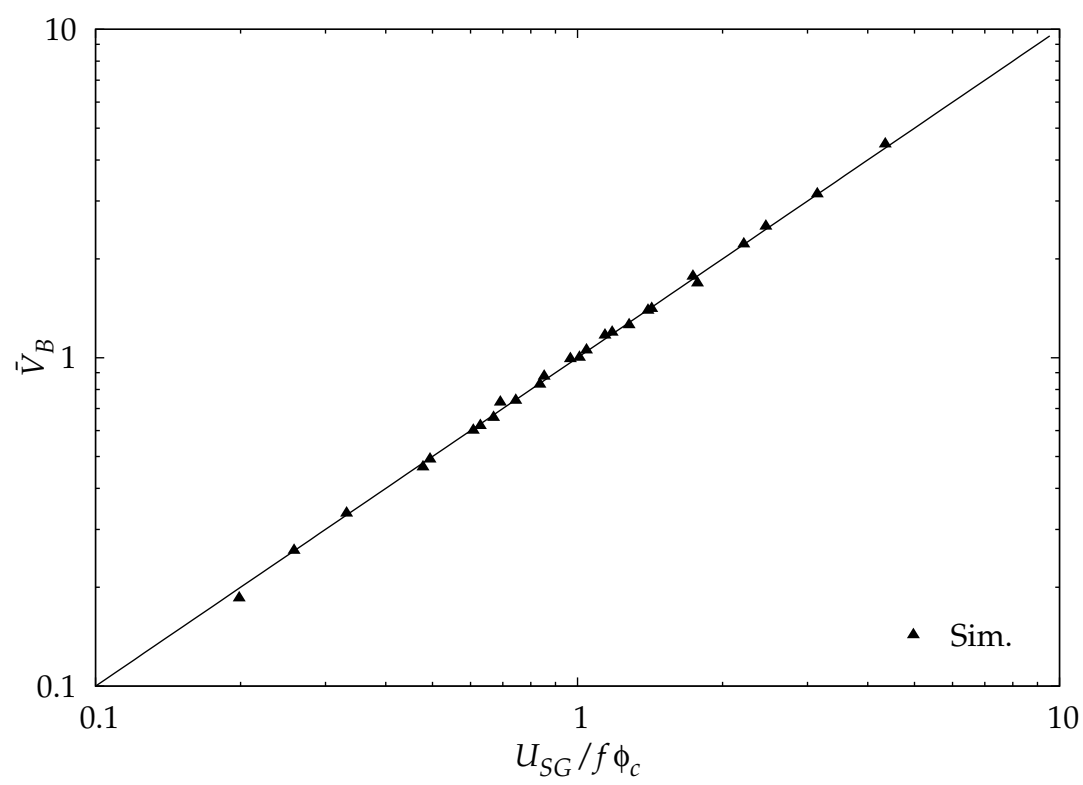

Figure 10: Normalised bubble volume as a function of $U_{S G} /\left(f \phi_{c}\right)$. Symbols: simulation data. Line: theoretical prediction given by Eq. 5

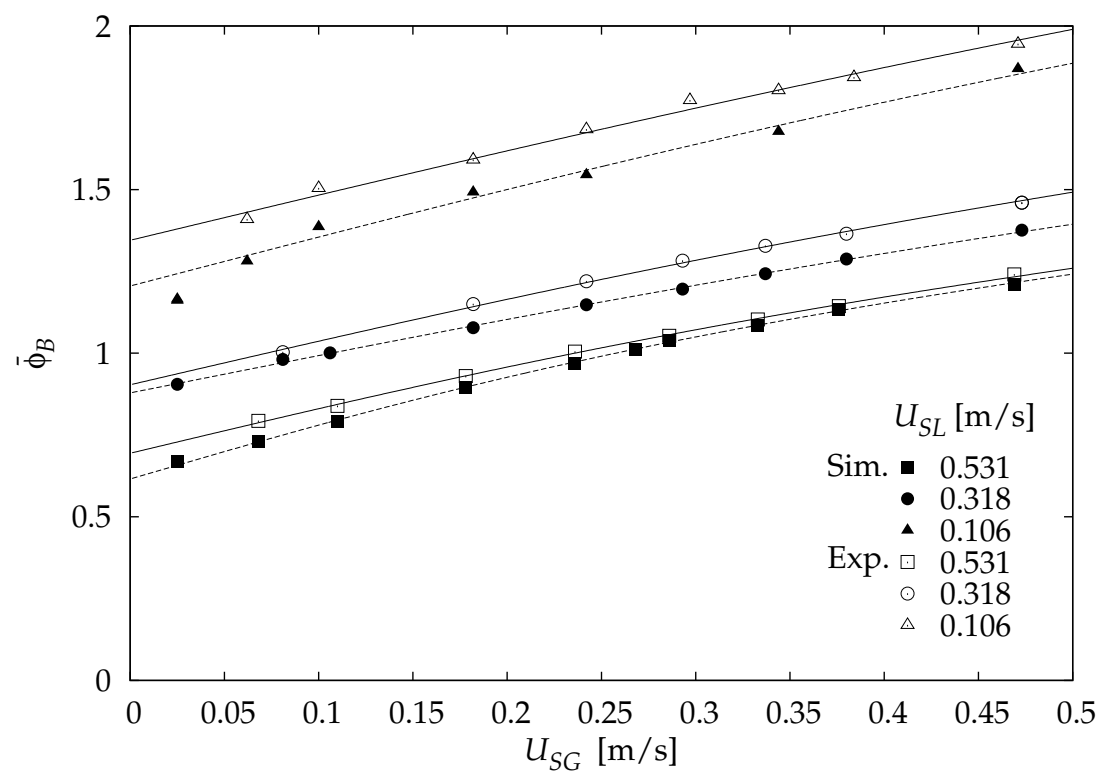

Figure 11: Normalised equivalent diameter $\bar{\phi}_{B}$ as a function of the gas superficial velocity $U_{S G}$. Empty symbols: experimental data, solid symbols: numerical data. Lines: solid and dashed lines, given by Eq. 6, correspond to experimental and numerical data, respectively.

In order to provide a quantitative comparison of the bubbles size, an equivalent diameter $\phi_{B}$ has been considered for each bubble. $\phi_{B}$ is estimated by matching the bubble volume predicted by Eq. 5 to the volume of a sphere $V_{S}=\pi \phi_{B}^{3} / 6$. Scaling $V_{S}$ with $A \phi_{c}$, the non-dimensionless 
volume of the sphere can be written as $\bar{V}_{S}=2 \bar{\phi}_{B}^{3} / 3$. The equivalent diameter is normalised with the capillary diameter and is then computed as the result of matching $\bar{V}_{B}$ and $\bar{V}_{S}$ :

$$
\bar{\phi}_{B}=\left(\frac{3}{2} \frac{U_{S G}}{f \phi_{c}}\right)^{1 / 3}
$$

Figure 11 shows the non-dimensional equivalent diameter as a function of the gas superficial velocity. Differences in equivalent diameter between experimental and numerical results range from $1.1 \%$ to $9.1 \%$ with an average of $5.2 \%$ difference. The equivalent diameter is estimated using Eq. 6 in both cases. Lines correspond to Eq. 6 , in which the prediction provided by Eq. 4 was included. Both experimental and numerical data correctly agree with the theoretical prediction. The equivalent diameter is found to be slightly smaller in numerical simulations than those from experiments, which coherently corresponds to the bigger values in $U_{G}$ and $f$ values from the simulations. It can be observed that increasing the liquid superficial velocity causes the reduction of the equivalent diameter. The value $\bar{\phi}_{B}=1$ allows for the distinction between the bubble $\left(\bar{\phi}_{B}<1\right)$ and the slug flow regime $\left(\bar{\phi}_{B}>1\right)$ in Fig. 11 .

\section{CONCLUSIONS}

- The first part of this work has presented a study on the numerical simulation of bubbles generated in a 3D T-junction (1 mm internal diameter capillaries), with an air/water mixture, using the commercial CFD solver ANSYS Fluent v15.0.7.

- Numerical results were compared with experimental data, both cases following the same conditions.

- Several trains of bubbles were simulated with high regularity and small dispersion in size during the bubble formation process.

- No artificial pinch-off mechanism was required for the detachment of bubbles.

- Bubble or slug flow regimes were obtained in all cases, both in experiments and numerical simulations. Only short and middle Taylor bubbles were generated in the slug flow pattern.

- Numerical simulations satisfactorily reproduced the formation of bubbles, both in the final shape as well as in the evolution of shape during the formation of bubbles.

- Several parameters were compared between 3D-CFD results and experimental data, such as the bubble velocity, void fraction, bubble generation frequency, Strouhal number and the bubble equivalent diameter.

- Numerical results were found to be of the same order of magnitude, though CFD simulations predict larger frequency, resulting in faster and smaller sized bubbles.

- Future work should focus in studying into more details the pinch-off process in the numerical simulation, in order to try to further improve these results.

- 3D-CFD simulations allowed to study new zones of interest, especially at low gas flow rate, and new parameters such as the bubble volume, which were not available from experimental results, showing that this is a useful tool to improve our current experimental knowledge on two-phase flows in minichannels. 


\section{Acknowledgments}

This work has been financially supported by the Spanish Ministerio de Economía y Competitividad, Secretaría de Estado de Investigación, Desarrollo e Innovación (Project numbers AYA2012-34131 and MTM2013-46313-R) and the Generalitat de Catalunya (Grant number 2014-SGR-1471).

\section{REFERENCES}

[1] S. Arias, D. Legendre D., R. González-Cinca, Numerical simulation of bubble generation in a T-junction. Computers \& Fluids, 56, 49-60, 2012.

[2] K. Yamamotoa, S. Ogata, Effects of T-junction size on bubble generation and flow instability for two-phase flows in circular microchannels. Int. J. Multiphase Flow, 49, 24-30, 2013.

[3] K. Yamamotoa, S. Ogata, Drag reduction of slug flows in microchannels by modifying the size of T-junctions. Int. J. Multiphase Flow, 62, 67-72, 2014.

[4] D. Qian, A. Lawal, Numerical study on gas and liquid slugs for Taylor flow in a Tjunction microchannel. Chem Eng Sci, 61(23), 7609-7625, 2006.

[5] M.N. Kashid, F. Platte, D.W. Agar, S. Turek, Computational modelling of slug flow in a capillary microreactor. J Comput Appl Math, 203(2), 487-497, 2007.

[6] M. De Menech, P. Garstecki, F. Jousse, H.A. Stone, Transition from squeezing to dripping in a microfluidic T-shaped junction. J Fluid Mech, 595, 141-161, 2008.

[7] I.L. Ngo, T.D. Dang, C. Byon, S.W. Joo, A numerical study on the dynamics of droplet formation in a microfluidic double T-junction. Biomicrofluidics, 9, 024107, 2015.

[8] S. Malekzadeh, E. Roohi, Investigation of Different Droplet Formation Regimes in a Tjunction Microchannel Using the VOF Technique in OpenFOAM. Microgravity Sci. Technol., 27, 231-243, 2015.

[9] Y. Shi, G.H. Tang, H.H. Xia, Lattice Boltzmann simulation of droplet formation in Tjunction and flow focusing devices. Computers \& Fluids, 90, 155-163, 2014

[10] S. Ben Saad, C. Gentric, J.F. Fourmigué, P. Clément, J.P. Leclerc, CFD and experimental investigation of the gas-liquid flow in the distributor of a compact heat exchanger. Chemical Engineering Research \& Design, 92(11), 2361-2370, 2014.

[11] S. Arias, X. Ruiz, L. Ramírez-Piscina, J. Casademunt, R. González-Cinca R., Experimental study of a microchannel bubble injector for microgravity applications. Microgravity Sci. Technol, 21, 107-111, 2009.

[12] S. Arias, R. González-Cinca, X. Ruiz, L. Ramírez-Piscina, J. Casademunt, Characterization of the performance of a minibubble generator in conditions relevant to microgravity. Colloids and Surfaces A: Physicochem. Eng. Aspects, 365, 52-55, 2010.

[13] M. Suo, P. Griffith, Two-phase flow in capillary tubes. J. Basic Eng., 86, 576-582, 1964. 
[14] K.S. Rezkallah, Weber number based flow-pattern maps for liquid-gas flows at microgravity. Int. J. Multiphase Flow, 22(6), 1265-1270, 1996.

[15] A.E. Dukler, J.A. Fabré, J.B. McQuillen, R. Vernon, Gas-liquid flow at microgravity conditions: flow patterns and their transitions. Int. J. Multiphase Flow, 14, 389-400, 1988.

[16] ANSYS® Academic Research, Release 15.0,7, Help System, ANSYS, Inc, 2014.

[17] D.L. Youngs, Time-Dependent Multi-Material Flow with Large Fluid Distortion. $K$. $W$. Morton and M. J. Baines, editors, Numerical Methods for Fluid Dynamics, Academic Press, 1982

[18] J.U. Brackbill, D.B. Kothe, C. Zemach, A Continuum Method for Modeling Surface Tension. J. Comput. Phys., 100, 335-354, 1992.

[19] C. Colin, J. Fabré, A.E. Dukler, Gas-liquid flow at microgravity conditions - I Dispersed bubble and slug flow. Int. J. Multiphase Flow, 17, 533-544, 1991.

[20] C. Colin, J. Fabré, Gas-liquid pipe flow under microgravity conditions: influence of tube diameter on flow pattern and pressure drops. J. Adv. Space Research, 16, 137-142, 1995.

[21] G. Puccetti, M. Tosi, B. Pulvirenti, G.L. Morini, Flow patterns of an air-water mixture at the exit of a micro T-junction. Experimental Thermal and Fluid Science, 67, 62-69, 2015.

[22] J. Carrera, X. Ruiz, L. Ramírez-Piscina, J. Casademunt, M. Dreyer, Generation of a monodisperse microbubble jet in microgravity. AIAA Journal, 46(8), 2010-2019, 2008.

[23] T. Fu, Y. Ma, D. Funfschilling, C. Zhu, H.Z. Li, Squeezing-to-dripping transition forbubble formation in a microfluidic T-junction. Chem Eng Sci, 65(12), 3739-3748, 2010.

[24] N. Zuber, J. Findlay, Average Volumetric Concentration in Two-Phase Systems. Trans ASME Jul Ht Transfer, 87, 453-468, 1969.

[25] J. McQuillen, C. Colin, J. Fabre, Ground-based gas-liquid flow research in microgravity conditions: state of knowledge. Space Forums, 3, 165-203, 1998. 